\title{
Pertussis Surveillance in Canada: Trends to 2012
}

\author{
Smith $\mathrm{T}^{1 *}$, Rotondo $\mathrm{J}^{1}$, Desai $\mathrm{S}^{1}$ and Deehan $\mathrm{H}^{1}$.
}

1 Public Health Agency of Canada, Center for Immunization and Respiratory Infectious Diseases, Ottawa,ON

* Corresponding author: tiffany.smith@phac-aspc.gc.ca

\section{Abstract}

Objective: The purpose of this report is to provide a summary of the pertussis activity in Canada.

Methods: A descriptive analysis of pertussis incidence by year, age group, gender and province/territory was conducted using national surveillance data, clinical administrative data and vital statistics data.

Results: Pertussis is an endemic cyclical disease in Canada with peaks in activity occurring every 2 to 5 years. Canada has experienced a decline in pertussis activity following the introduction of routine pertussis immunization programs. The incidence of pertussis is highest in infants and children. Hospitalization and mortality are more common among infants, particularly those less than three months of age. Trends in pertussis vary by province and territory. Canada experienced a notable increase in incidence in 2012. Reasons for this increase are unknown.

Conclusion: Our understanding of the epidemiology of pertussis in Canada could be enhanced by improved approaches for monitoring the disease. Although the peak in activity observed in 2012 could be an isolated event, further work to support outbreak response in provinces and territories, including rapid research tools and resources, should be considered.

\section{Introduction}

In Canada, pertussis has been under national surveillance since 1924 and a target of childhood immunization programs since 1943. Infant, child, adolescent, and adult routine immunization programs exist across Canada, with notable differences in the vaccine product used and schedule of immunization, depending on the province/territory ${ }^{1}$. The introduction of childhood immunization programs contributed to a significant reduction in disease; however, pertussis remains one of the most commonly reported vaccine preventable diseases in Canada. In early 2012, the Public Health Agency of Canada (the Agency) received reports of increased pertussis activity in multiple jurisdictions which prompted a review of Canadian pertussis data. The purpose of this report is to provide a summary of pertussis activity in Canada since it became notifiable with an emphasis on more recent activity. 


\section{Methods}

\section{DATA SOURCES}

\section{National Case Reports}

Nationally reported confirmed cases of pertussis from 1924 to 2011 were extracted from the Canadian Notifiable Diseases Surveillance System (CNDSS) database in March $2013^{2}$. National pertussis case definitions were published in $1991^{3}, 2000^{4}$ and $2009^{5}$. Prior to 2000 , a confirmed case of pertussis required isolation of Bordetella pertussis and presence of clinically compatible symptoms. Between 2000 and 2008 the case definition required laboratory confirmation (via culture from an appropriate clinical specimen or detection of DNA) or an epidemiological link to a laboratory confirmed cases and one of a list of clinical symptoms. In 2009, the case definition was further refined to require those cases in which $B$. pertussis DNA was detected to also have clinically compatible symptoms. Confirmed pertussis cases for 2012 were obtained directly from the provinces and territories in March 2013 by the Agency's Centre for Immunization and Respiratory Infectious Diseases, and are preliminary.

\section{Hospitalizations}

Pertussis cases reported through the Canadian Immunization Monitoring Program Active (IMPACT) from 1991 to 2012 were extracted from the IMPACT database in April $2013^{6}$. Data for 2012 are preliminary. IMPACT has provided enhanced data on pediatric pertussis cases treated at tertiary care centres in Canada to the Agency since 1991, initially from 5 centers and then expanded to 10 centers in 1993, 11 centers in 1995 and 12 centers in 1999.

Data on pertussis-related acute care hospitalizations from 1995 to 2010 were extracted from the Canadian Institute for Health Information's (CIHI) Hospital Morbidity Database (HMD) in June 2013. These dates were selected as the data sets were complete for each year. Pertussis hospitalizations were defined as those with the International Classification of Diseases (ICD) version 9 or 10 discharge diagnoses (all diagnosis levels) corresponding to pertussis $(0330,0339,4843, A 370$ or A379). Exclusion criteria included hospital transfers and readmissions, with readmissions defined as hospitalizations which occurred $<1$ year apart.

\section{Vital Statistics}

Mortality data were obtained from the Death Database, a national mortality database which includes demographic and medical (underlying cause of death) information collected annually from all provincial and territorial vital statistics registries on all deaths in Canada ${ }^{7}$. Deaths with an underlying cause of pertussis were identified using the same ICD codes listed above.

\section{Analysis}

A descriptive analysis of pertussis incidence by year, age group, gender and province/territory was conducted. Population data for the calculation of rates was obtained from Statistics Canada ${ }^{8}$. Data sent to CNDSS are aggregated by age group, therefore the calculation of age-specific rates were limited to the following age groups in years $<1,1-4,5-9,10-14,15-19,20-24,25-29,30-39,40-59$, and 60 and over. Confirmed case rates are given per 100,000 population. Provinces/territories that were not able to report case counts were removed from the denominator for the corresponding year. Annual percent change was used to describe changes in reporting rates over time. Incidence rate ratios were calculated to examine gender differences.

\section{Results}

\section{Annual Incidence}

In the five years prior to vaccine introduction, the average incidence of pertussis was 156 cases per 100,000 population. After vaccine introduction, the incidence decreased to a mean annual low of 7 cases per 100,000 population from 1984 to 1988 (Figure 1). A resurgence of pertussis was observed beginning in 1989/90 which peaked at 34.9 cases per 100,000 in 1994. After 1998, incidence declined to the lowest incidence recorded in Canada at 2 cases per 100000 in 2011. A seven-fold increase in national incidence to 13.9 per 100,000 was 
observed in 2012. Observing trends over time, peaks in activity occurred every 2 to 5 years. These peaks have become less obvious as incidence of disease has decreased.

Figure 1. Reported cases and incidence rate (per 100,000 population) of Pertussis in Canada by year, 1924 to $2012^{*}$

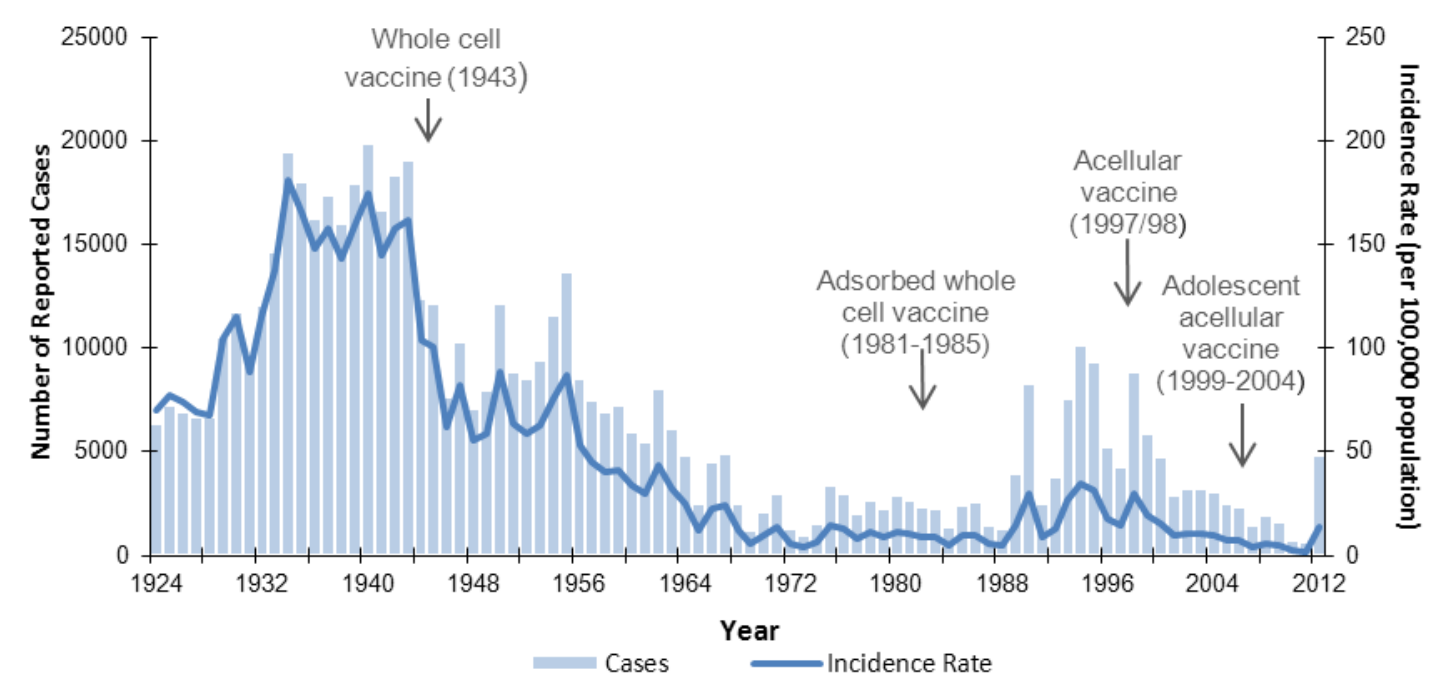

\begin{abstract}
*Case data from 1924 to 2011 were obtained from the Canadian Notifiable Diseases Surveillance System. Case data for 2012 were obtained directly from provinces and territories by CIRID and are preliminary. PEI did not report 1924-1928; Newfoundland did not report until 1949; Yukon did not report 1924-1955; Northwest Territories did not report 1924-1958; Nunavut data for 1999 are only partial, for 2007 \& 2009 are missing, and for 2008, 2010-2011 are preliminary. Population data (July 1st annual estimates) were obtained from Statistics Canada.
\end{abstract}

In general, trends in pertussis hospitalization data were similar to those observed in notifiable disease reports (Figure 2). National case reports and acute care hospitalizations decreased by 92\% from 1995 to 2010; case reports from 9308 (31.8 per 100,000) in 1995 to 748 (2.2 per 100,000) in 2010, and HMD hospitalizations from $2,016(6.8$ per 100,000$)$ in 1995 to $168(0.5$ per 100,000$)$ in 2010. In this same time frame, IMPACT hospitalizations decreased by $82 \%$ from 264 in 1995 to 48 in 2010. Peaks in activity in 1998 and minor increases in 2002 and 2008 were observed across all data sources. However, minor increases in hospital admissions were also observed in 2004 (HMD: 16\%; IMPACT: 65\%) and 2009 (HMD: 20\%; IMPACT: 26\%) while case reports declined in these years from the preceding year by $4 \%$ and $18 \%$ respectively. As with national case reports, cases reported through IMPACT hospitals increased in 2012, but not as dramatically as the national incidence rate of disease (2-fold increase). 
Figure 2. Number of reported cases and hospitalizations for pertussis in Canada by year, 1995 to $2012^{*}$

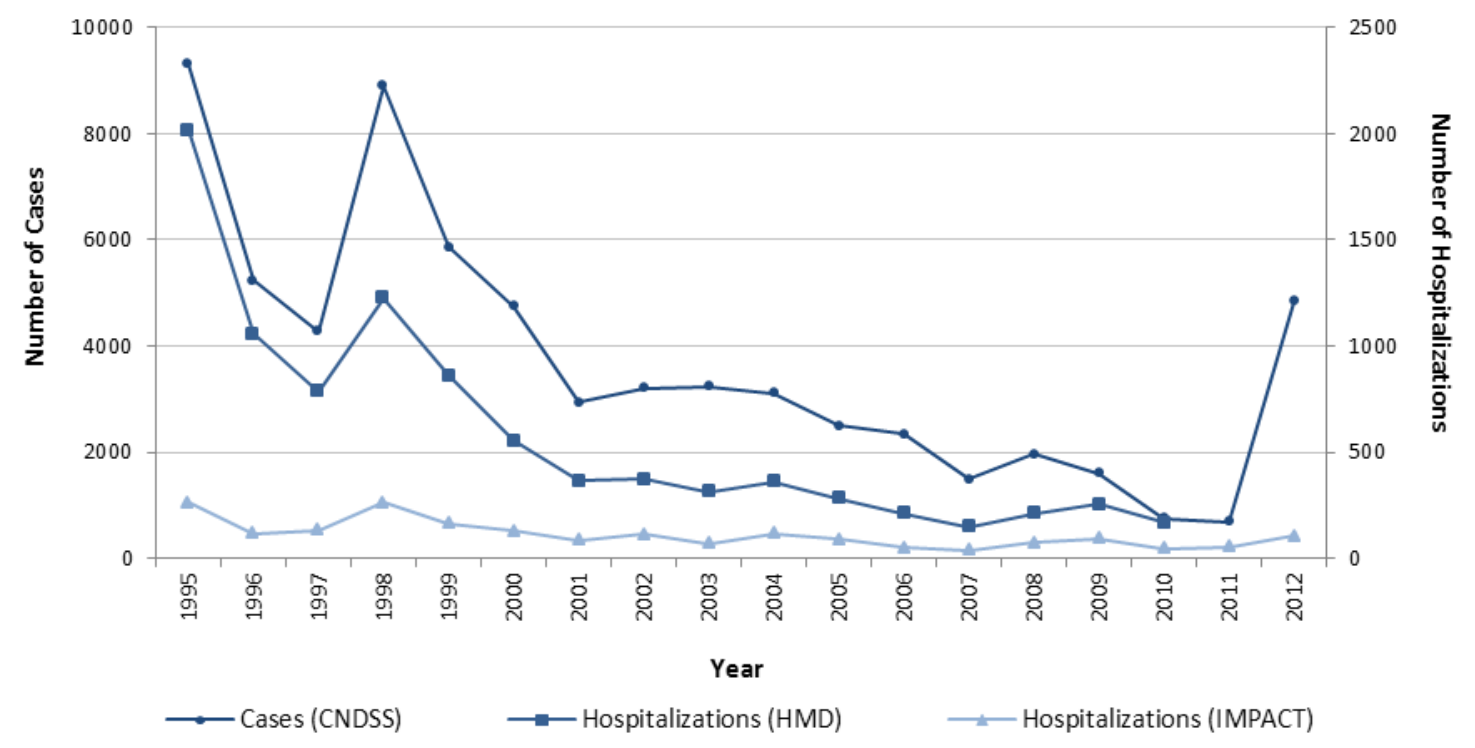

*Case data from 1995 to 2011 were obtained from the Canadian Notifiable Diseases Surveillance System. Case data for 2012 were obtained directly from provinces and territories by CIRID and are preliminary. Nunavut data for 1999 are only partial, for 2007 \& 2009 are missing, and for 2008, 2010-2011 are preliminary; IMPACT data for 2012 are preliminary.

\section{Age Distribution}

The incidence of pertussis is highest in infants and children, and decreases sharply in those older than 14 years (Figure 3). The highest mean incidence rates from 2005 to 2011 were 72.2 cases per 100,000 population among infants less than 1 year of age (mean: 261 cases per year), 25.6 cases per 100,000 population among 1 to 4 year olds (mean: 362 cases per year), and 16.0 cases per 100,000 population among 10 to 14 year olds (mean: 328 cases per year). Comparatively, the mean incidence of pertussis among all individuals 15 years and greater from 2005 to 2011 was 1.6 cases per 100,000 population (mean: 428 cases per year).

Between 2005 and 2011, the incidence of pertussis decreased in all age groups, most notably among those aged 10 to14 years (84\% decrease) and those aged 15 to 19 years of age (81\% decrease). In 2012, increases in incidence were observed across all age groups nationally with the highest incidence rates in those less than one year (120.8 per 100,000; $n=460$ ) and those 10 -14 years of age (64.1 per 100,$000 ; n=1203)$. The highest incidence rates in the majority of provinces/territories were within age groups below 15 years of age. 
Figure 3. Incidence rate (per 100,000 population) of pertussis reports in Canada by age group (in years) and year, 1980 to $2012^{*}$

${ }^{*}$ Case data from 1980 to 2011 were obtained from the Canadian Notifiable Diseases Surveillance System. Case data for

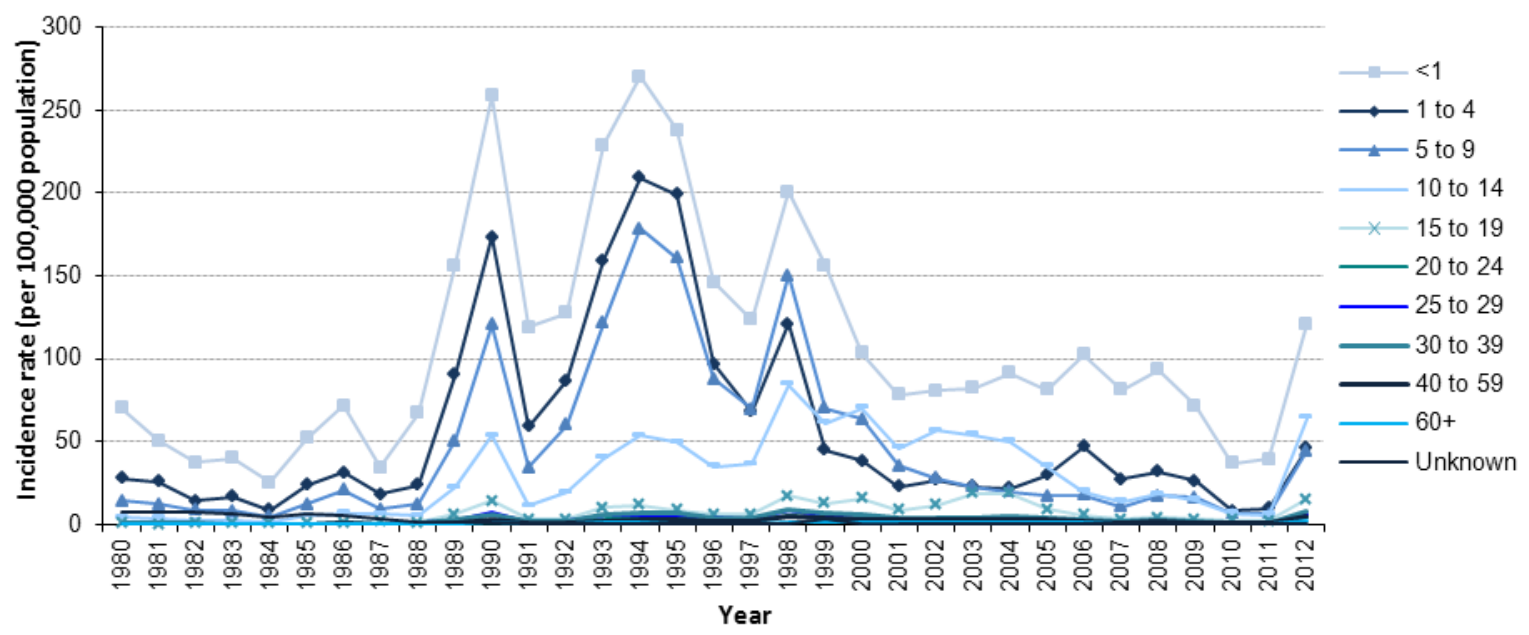

2012 were obtained directly from provinces and territories by CIRID and are preliminary. Nunavut data for 1999 are only partial, for $2007 \& 2009$ are missing, and for 2008, 2010-2011 are preliminary. Population data (July 1st annual estimates) was obtained from Statistics Canada and uses.

Fluctuations in the proportion of pertussis cases by age group have occurred in the last twenty years, particularly among children aged 1 to 9 years and adolescents aged 10 to 19 years (Figure 4). During the 1990s, the highest proportion of cases occurred in the 1 to 9 year age groups (mean: 62\% per year). Between 2000 and 2005, the proportion of cases in adolescent age groups (i.e. 10 to 19 years) increased and was on average $42 \%$ of cases per year, while the proportion of cases among those 1 to 9 decreased to an average of $30 \%$ per year. Between 2006 and 2011, cases 10 to 19 years of age decreased (mean: 23\% per year), while the number of cases 1 to 9 years increased marginally (mean: $38 \%$ per year). As these larger shifts occurred, minor increases occurred in infants ( $<1$ year) and adults (20 years and older) from respective annual means of $12 \%$ and $10 \%$ of cases in the 1990 s, to $18 \%$ and $21 \%$ of cases between 2006 and 2011. In 2012, $10 \%$ of cases were less than one, $32 \%$ were between 1 to 9 years, 31\% were between 10 and 19 years and $27 \%$ were 20 years or older.

In contrast, the age distribution of acute care hospitalized cases has not varied considerably over time with the proportion of hospitalizations consistently highest among children less than one. Between 1995 and 2010, on average each year, a total of $69 \%$ of pertussis-related admissions were among those less than 1 (range: 59 to $78 \%$ ). Of those less than one, the majority of admissions occurred in young infants with an annual mean of $62 \%$ cases under the age of 3 months (range: 49 to $71 \%$ ). 
Figure 4. Age distribution of pertussis cases in Canada, 1990 to 2012*

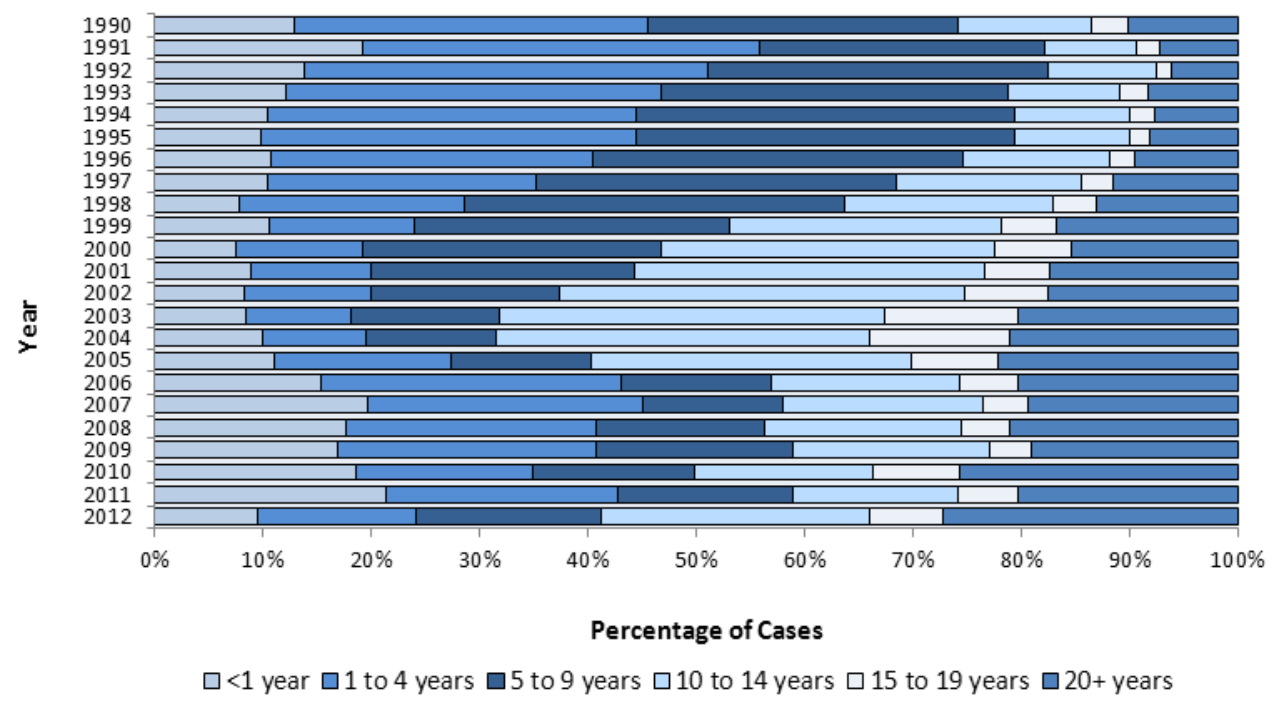

${ }^{*}$ Case data from 1990 to 2011 were obtained from the Canadian Notifiable Diseases Surveillance System. Case data for 2012 were obtained directly from provinces and territories by CIRID and are preliminary. Nunavut data for 1999 are only partial, for $2007 \& 2009$ are missing, and for 2008, 2010-2011 are preliminary.

\section{Gender Distribution}

From 1990 to 2012, the number of nationally reported pertussis cases and incidence rate has been consistently higher among females in all age groups. During this time period, the annual median male to female ratio in incidence rate was 0.87:1 (range: $0.81: 1$ to $0.96: 1$ ). Females made up on average $54.0 \%$ of cases, males $45.8 \%$ of cases, and $0.2 \%$ had an unknown gender each year. Similarly, between 1995 and 2010, the median male to female ratio in the incidence rate of hospitalization was 0.89:1 (range: 0.73:1 to 1.05:1) and on average, each year, females made up $53.5 \%$ of cases.

\section{Geographic Distribution}

Asynchrony in the timing and geographic focus of 2-5 year cyclical pertussis peaks is expected. Although Canada experienced an overall decline in pertussis incidence from the late 1990s to 2011, the timing, magnitude and overall trend in decreased activity varied across the provinces and territories (Figure 5). From 1995 to 2011, peaks in laboratory-confirmed pertussis notifications were observed, with the highest incidence by province across this period variously occurring in 1995 (Manitoba, Ontario, New Brunswick and Nova Scotia), 1996 (Alberta), 1997 (Prince Edward Island), 1998 (Quebec), 1999 (Saskatchewan, Newfoundland, Northwest Territories and Nunavut) or 2000 (British Columbia and Yukon). The absolute difference between the highest and lowest incidence rates within a province/territory during this time, ranged from 18 cases per 100,000 to a much larger difference of 489 cases per 100,000, with large fluctuations more common in jurisdictions with smaller populations. From provincial-/territorial-specific peaks to 2011, each province and territory experienced a decline of more than $75 \%$ in incidence with the vast majority seeing decreases exceeding $90 \%$.

National increases in incidence are typically the result of peaks observed in multiple jurisdictions. The national increase observed in 1998 was due to increased activity experienced in Manitoba, Ontario, Quebec and New Brunswick. This was also the case in 2012, when increases in incidence relative to 2011 were observed in 9 of the 13 provinces and territories. At the provincial/territorial level, these increases ranged from a negligible 0.7 cases per 100,000 to a more substantial 152 cases per 100,000. Within jurisdictions that experienced substantial increases, causes varied from province-wide outbreaks, to regional outbreaks, to localized outbreaks. The most notable increase in activity was observed in New Brunswick, due to a province-wide outbreak that accounted for approximately one-third of the national case count in 2012. 


\section{Mortality}

From 2000 to 2009, Statistics Canada reported a total of six deaths with pertussis listed as the underlying cause of death. Between zero and two deaths occurred each year and all deaths were reported in infants less than one year of age. However, this is likely an underestimation of the total deaths associated with pertussis in Canada. According to data from IMPACT, from 2000 to 2009, 11 of the 858 reported pertussis cases identified through IMPACT died (1.2\%). From 1991 to 2012, 30 deaths have been reported through IMPACT, with the number ranging from zero to four per year (Figure 6). The majority of deaths occurred in infants less than 2 month of age (78\%). All deaths, except for one, occurred in infants with no previous pertussis immunizations and $78 \%$ of deaths were in children considered previously healthy. Causes of death included respiratory distress/failure, pneumonia, hypoxia, pulmonary hypertension, pulmonary hemorrhage, fulminant septic shock, cardiovascular failure, and acute arrhythmia.

*Case data from 1995 to 2011 were obtained from the Canadian Notifiable Diseases Surveillance System. Case data for 2012 were obtained directly from provinces and territories by CIRID and are preliminary. Nunavut data for 1999 are only partial, for $2007 \& 2009$ are missing, and for 2008, 2010-2011 are preliminary; Population data (July 1st annual estimates) were obtained from Statistics Canada.
Figure 5. Trends in annual incidence of pertussis (per 100,000 population) by province/territory, 1995 to 2012

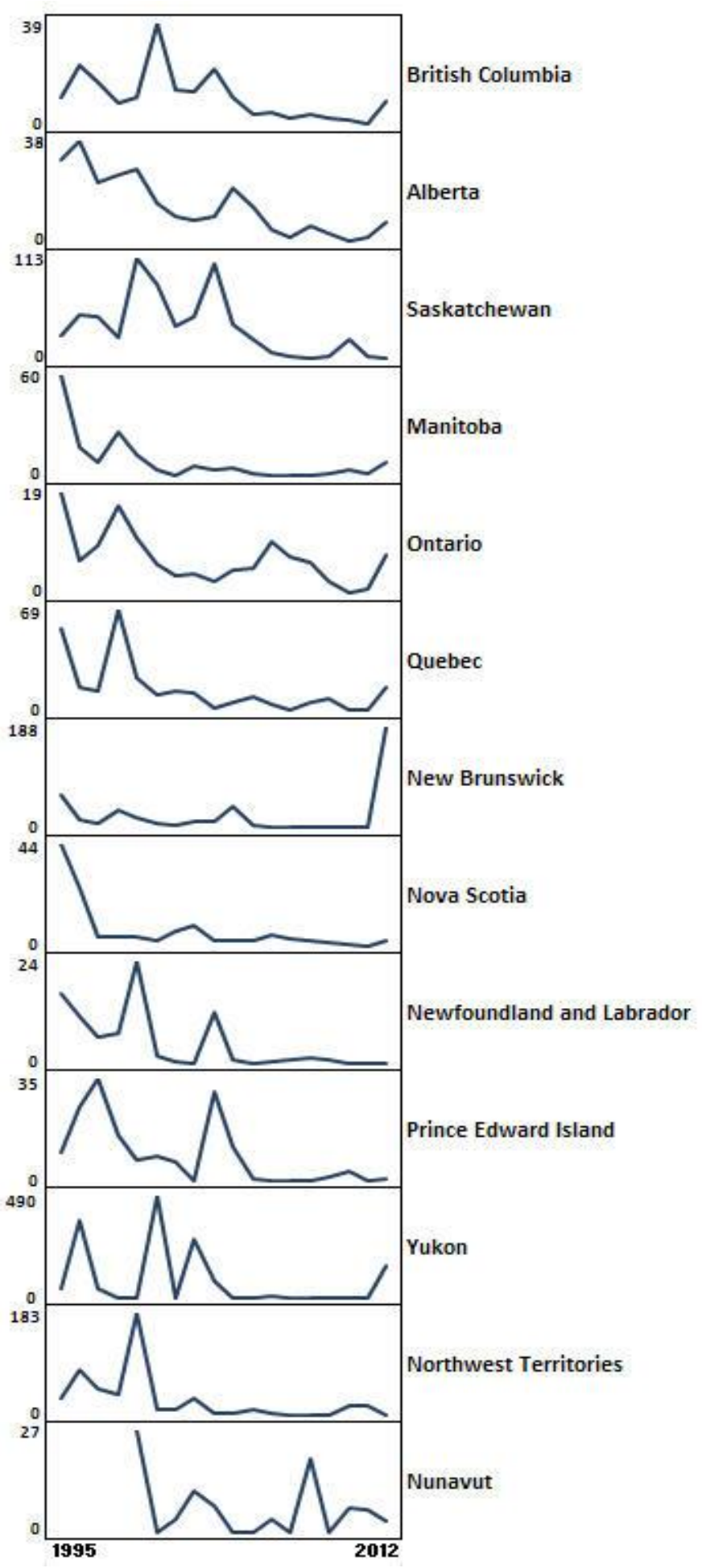


Figure 6. The number of pertussis cases and deaths, IMPACT, 1991 to $2012^{*}$

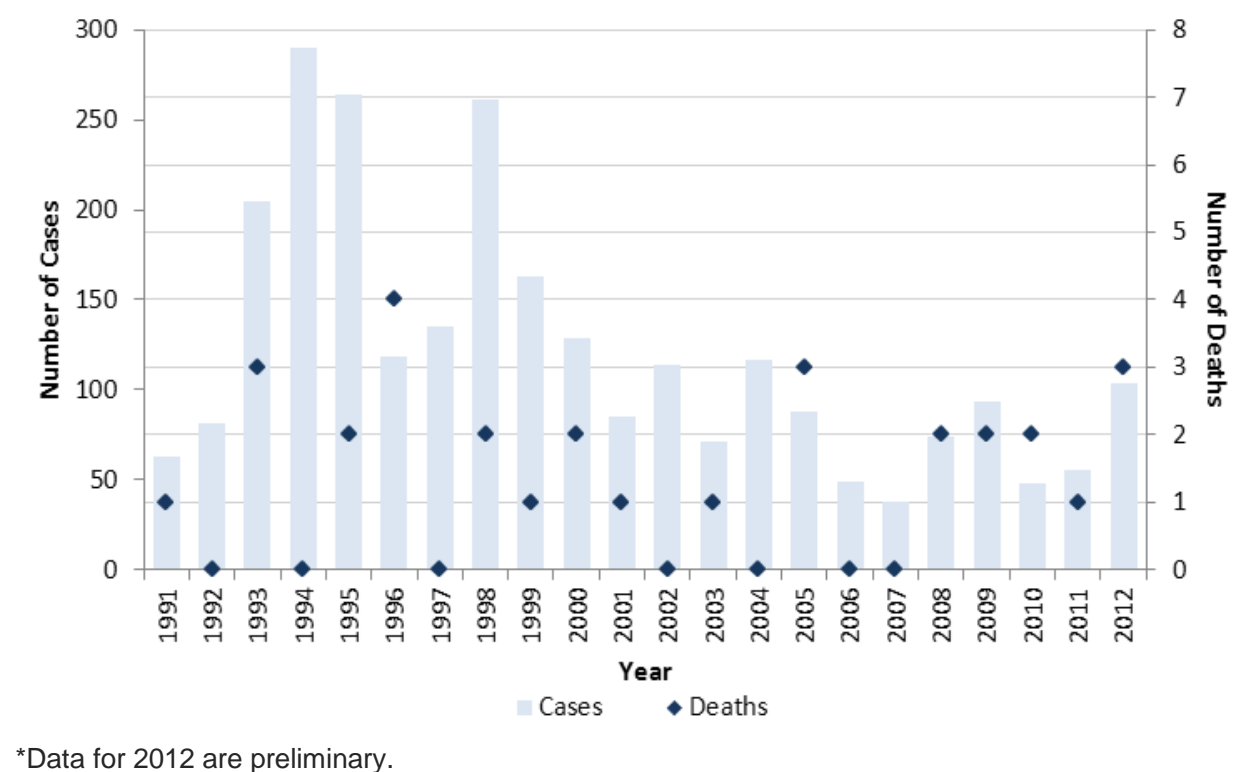

\section{Discussion}

Pertussis is an endemic cyclical disease in Canada with peaks in activity occurring every 2 to 5 years. Although resurgence was observed in the 1990s, overall, Canada has experienced a decline in pertussis activity following the introduction of routine pertussis immunization programs. This was first observed with the introduction of the whole cell vaccine in 1943, followed by the introduction of the acellular vaccine in 1997/98 and lastly, between 2005 and 2011 after the introduction of adolescent immunization programs across the country ${ }^{9}$. As previously noted, the resurgence observed in the 1990s was likely due to a combination of factors, including the low efficacy of the whole-cell vaccine introduced between 1981 and 1985, as well as increased physician awareness and improved diagnostics and reporting of pertussis infection ${ }^{10}$. From a geographic perspective, all provinces and territories experienced a decline in the incidence in pertussis in the seven years prior to 2012; however, trends in disease were unique to each jurisdiction in terms of timing and magnitude of cyclical peaks.

The incidence of pertussis is consistently highest among infants, as are hospitalization and death, particularly among those 3 months of age or less. This group is of particular interest as they are too young to benefit directly from immunizations. Different approaches have been tried which include cocooning and immunization of pregnant women to allow for passive immunity to the infant ${ }^{11,12,13}$. Canada's National Advisory Committee on Immunization has recommended immunization in pregnancy if there is a regional outbreak of pertussis ${ }^{14}$.

The increase in incidence observed in 2012 was due to outbreaks in several jurisdictions across the country. Increases in incidence were observed across all age groups nationally with the highest incidence rates in those less than one. However, age groups most affected varied by province/territory. As with national case reports, cases reported through IMPACT hospitals increased in 2012, but not as dramatically which could suggest that the increase in cases was not associated with increasing severity.

Increases in pertussis activity have also recently occurred in other countries with several reasons for increases proposed in the literature, including waning immunity ${ }^{15}$, reduced natural immune boosting ${ }^{16}$, higher rates of detection $^{17}$, genetic changes in the bacteria ${ }^{18}$, as well as clustering of unvaccinated individuals ${ }^{19}$. Based on nationally available data, it's difficult to determine the contributing factors for the increases observed in Canada in 2012. At present, data collected at the national level is minimal, often in an aggregate format that does not include the immunization history of cases. The National Microbiology Laboratory does not currently conduct routine surveillance of circulating pertussis strains. According to the validated results of the 2009 childhood National Immunization Coverage Surveys, immunization coverage is high; $98 \%$ for four or more doses in two year olds and 
$89 \%$ for five or more doses among seven year olds ${ }^{20}$. However, evaluations of specific immunization programs and coverage within populations and sub-populations in Canada have yet to be done in a systematic way.

\section{Limitations}

Due to the passive nature of CNDSS, reported cases are expected to be underestimates of the true burden of disease, particularly among adolescents and adults. It is likely that the incidence of pertussis is actually higher among adults than reported, as symptoms are generally milder, and testing for $B$. pertussis among adults with persistent cough is infrequent. In addition, the pertussis-related hospitalizations identified in the HMD are coded based on the physician's diagnosis as it appears in the medical chart and do not necessarily match the national case definition (e.g. may not be based on laboratory results). As a result, the number of pertussis-related acute care hospitalizations featured in this report could be an overestimate of the actual burden.

\section{Conclusion}

In conclusion, our understanding of the epidemiology of pertussis in Canada could be enhanced by improved approaches for monitoring the disease. National pertussis surveillance that also captures vaccine history, microbiological characteristics and indication of outbreaks, may yield answers that can inform public health action. Although the peak in activity observed in 2012 could be an isolated event, further work to support outbreak response in provinces and territories, including rapid research tools and resources, should be considered.

\section{References}

1. Public Health Agency of Canada. Publicly funded Immunization Programs in Canada - Routine Schedule for Infants and Children including special programs and catch-up programs (as of March 2013). http://www.phac-aspc.gc.ca/im/ptimprog-progimpt/table-1-eng.php

2. Public Health Agency of Canada. National Notifiable Diseases. http://www.phac-aspc.gc.ca/aids-sida/about/dis-eng.php.

3. Health and Welfare Canada. Canadian Communicable Disease Surveillance System: disease-specific case definitions and surveillance methods. Can Dis Wkly Rep 1991;17(S3).

4. Health Canada. Case definitions for diseases under national surveillance. CCDR. 2000;26(S3).

5. Public Health Agency of Canada. Case definitions for communicable diseases under national surveillance. CCDR. 2009; 35(S2).

6. Canadian Paediatric Society. Canadian Immunization Monitoring Program Active. http://www.cps.ca/en/impact.

7. Canadian Vital Statistics - Death Database, Statistics Canada, Health Statistics Division, [2000 to 2009]. Accessed 2013 July 15.

8. Canadian Vital Statistics - July Provincial Population Estimates, Statistics Canada, Health Statistics Division, [1924-2005 (final intercensal estimates); 2006-09 (final postcensal estimates); 2010-11 (updated postcensal estimates); 2012 (preliminary postcensal estimates)]. Accessed 2013 July 15.

9. National Advisory Committee on Immunization. Prevention of pertussis in adolescents and adults. CCDR. 2003; 29(ACS5).

10. Health Canada. National Consensus Conference on Pertussis. CCDR. 2003; 29(S3):1-33.

11. Wiley KE, Zuo Y, Macartney KK, McIntyre PB. Sources of pertussis infection in young infants: a review of key evidence informing targeting of the cocoon strategy. Vaccine. 2013;31(4):618-25.

12. Healy CM, Rench MA, Baker CJ. Importance of timing of maternal combined tetanus, diphtheria, and acellular pertussis (Tdap) immunization and protection of young infants. Clin Infect Dis. 2013;56(4):539-44.

13. Hardy-Fairbanks AJ, Pan SJ, Decker MD, Johnson DR, Greenberg DP, Kirkland KB, et al. Immune responses in infants whose mothers received tdap vaccine during pregnancy. Pediatr Infect Dis J. 2013;32(11):1257-60. 
14. Canadian Immunization Guide [web page]. Pertussis Vaccine. http://www.phac-aspc.gc.ca/publicat/cig-gci/p04-pert-coqu-eng.php.

15. Klein NP, Bartlett J, Rowhani-Rahbar A, Fireman B, Baxter R. Waning protection after fifth dose of acellular pertussis vaccine in children. N Engl J Med. 2012;367 (11):1012-1019.

16. Lavine JS, King AA, Bjørnstad ON. Natural immune boosting in pertussis dynamics and the potential for longterm vaccine failure. Proc Natl Acad Sci U S A. 2011;108(17):7259-64.

17. Cherry JD. Epidemic pertussis in 2012 - the resurgence of a vaccine-preventable disease. N Engl J Med. 2012;367(9):785-787.

18. Mooi FR, VAN DER Maas NA, De Melker HE. Pertussis resurgence: waning immunity and pathogen adaptation - two sides of the same coin. Epidemiol Infect. 2013 Feb 13:1-10.

19. Atwell JE, Van Otterloo J, Zipprich J, Winter K, Harriman K, Salmon DA et al. Nonmedical vaccine exemptions and pertussis in California, 2010. Pediatrics. 2013;132(4):624-30.

20. Laroche J, Gendron MP, Abdel-Motagally M. Impact of validating respondent responses on national immunization coverage estimates [poster]. Canadian Immunization Conference. 2012 Dec.

\section{Notes and Acknowledgements}

Vital statistics data were provided to the Agency by Statistics Canada with the knowledge and consent of the Provincial and Territorial Vital Statistics Registrars whose cooperation is gratefully acknowledged. Although parts of this report are based on data provided by $\mathrm{ClHI}$, the analyses, conclusions, opinions and statements expressed herein are those of the Agency, and not necessarily those of CIHI. Provincial/territorial estimates produced by the Agency may differ from those published by provinces/territories due to variations in data used at the time of analysis. We gratefully acknowledge the participation of health care providers, public health officials and laboratories across the country in the routine reporting of pertussis. We would also like to thank all who participated in the development of the report, including: Vicky Springman (PHAC), Monique St-Laurent (PHAC), John Spika (PHAC), Julie Bettinger (IMPACT), Scott Halperin (IMPACT), Danuta Skowronski (British Columbia), Monika Naus (British Columbia), Theresa St. Jean (Alberta), Kimberley Simmonds (Alberta), Helen Bangura (Saskatchewan), Valerie Mann (Saskatchewan), Patricia Caetano (Manitoba), Shelley Deeks (Ontario), Jastej Dhaliwal (New Brunswick), Carolyn Sanford (Prince Edward Island), Anne Neatby (Prince Edward Island), Cathy O'Keefe (Newfoundland and Labrador), Gillian Butler (Newfoundland and Labrador), Beverly A. Billard (Nova Scotia), Lori Strudwick (Yukon), Karolina Machalek (PHAC/Yukon), Heather Hannah (Northwest Territories), Bryany Denning (Northwest Territories), Carolina Palacios (Nunavut), and Angie Mullen (Nunavut).

\section{Conflict of interest}

No conflicts of interests to declare.

\section{Funding}

None 